\title{
Nearly Conformal Composite Higgs Model
}

\author{
Thomas Appelquist, ${ }^{1}$ James Ingoldby $\odot,{ }^{2}$ and Maurizio Piai ${ }^{3}$ \\ ${ }^{1}$ Department of Physics, Sloane Laboratory, Yale University, New Haven, Connecticut 06520, USA \\ ${ }^{2}$ Abdus Salam International Centre for Theoretical Physics, Strada Costiera 11, 34151 Trieste, Italy \\ ${ }^{3}$ Department of Physics, College of Science, Swansea University, Singleton Park, Swansea, Wales SA2 8PP, United Kingdom
}

(Received 23 December 2020; revised 9 March 2021; accepted 12 April 2021; published 14 May 2021)

\begin{abstract}
We analyze a composite Higgs model based on the confining $S U(3)$ gauge theory with $N_{f}=8$ Dirac fermions in the fundamental representation. This gauge theory has been studied on the lattice and shown to be well described by a dilaton effective field theory (EFT). Here we modify the EFT by assigning standardmodel quantum numbers such that four of the composite pseudo-Nambu-Goldstone boson (pNGB) fields form the standard-model Higgs doublet, by coupling it to the top quark and by adding to the potential a term that triggers electroweak symmetry breaking. The model contains a pNGB Higgs boson, a set of heavier pNGBs, and an approximate dilaton in the same mass range. We study the phenomenology of the model and discuss the amount of tuning required to ensure consistency with current direct and indirect bounds on new physics, highlighting the role of the dilaton field.
\end{abstract}

DOI: 10.1103/PhysRevLett.126.191804

Introduction.-Lattice studies of the $S U(3)$ gauge theory with $N_{f}=8$ Dirac fermions in the fundamental representation show evidence of a light scalar singlet [1-5]. (Similar results hold with $N_{f}=2$ fermions in the symmetric representation [6-11].) The suggestion that this state might be a dilaton has fueled a revival of interest in the dilaton effective field theory (EFT). Its history dates back to dynamical symmetry breaking [12-14], well before this recent lattice-driven activity [15-26]. Existing lattice data, analyzed via the dilaton EFT [20,21], yielded the first measurement of a key, large anomalous dimension related to the fermion bilinear condensate [27]. The results are consistent with earlier expectations [28] and with recent high-loop perturbative studies $[29,30]$.

This theory, with a global $S U(8) \times S U(8)$ symmetry, broken to the diagonal $S U(8)$, is a natural candidate to build a composite Higgs model (CHM) [31-33] (see also [34-40] and references therein). Lattice studies of $S U(2)$ [41-47], $S U(4)$ [48-52], and $S p(4)$ [53-55] gauge theories have explored the possible origin of CHMs. The $S U(3)$ gauge theory has distinctive features: the presence of a light scalar singlet that modifies the EFT description of the CHM (see also Ref. [56]) and the presence of large anomalous dimensions. Furthermore, ordinary baryons can give rise to top compositeness [34].

Published by the American Physical Society under the terms of the Creative Commons Attribution 4.0 International license. Further distribution of this work must maintain attribution to the author(s) and the published article's title, journal citation, and DOI. Funded by SCOAP ${ }^{3}$.
In this Letter, we show that the presence of the dilaton field in the EFT allows us to construct an appealing CHM based on the $S U(8) \times S U(8) / S U(8)$ coset. We demonstrate that observables such as the ratio of the mass of the Higgs boson, $m_{h} \simeq 126 \mathrm{GeV}$, to the electroweak vacuum expectation value (VEV), $v \simeq 246 \mathrm{GeV}$, and to the mass of the additional heavy scalars, are substantially altered with respect to generic CHM expectations. We highlight how current lattice studies might already be exploring phenomenologically relevant regions of parameter space. These statements depend on the value of a (currently) unknown scaling dimension $w$, which in principle can be measured on the lattice.

The model.-We assign to the eight Dirac fermions the quantum numbers indicated in Table I. The global $S U(8) \times S U(8)$ symmetry group is broken both explicitly (by a diagonal mass term) and spontaneously (by the strong dynamics) to its diagonal $S U(8)$ subgroup. The gauge

TABLE I. Quantum number assignments of the Dirac fermions. $S U(3)_{C} \times S U(2)_{L} \times U(1)_{Y}$ is the standard model (SM) gauge group, while $S U(3)$ is the strongly coupled gauge symmetry. We denote with $\alpha=1,2$ the $S U(2)_{L}$ index. The fermions denoted by $R_{1,2}$ form a fundamental representation of the global $S U(2)_{R}$ custodial symmetry. A model with similar assignments has been considered in Ref. [34].

\begin{tabular}{lcccc}
\hline \hline Fermion & $S U(2)_{L}$ & $U(1)_{Y}$ & $S U(3)_{c}$ & $S U(3)$ \\
\hline$L_{\alpha}$ & 2 & 0 & 1 & 3 \\
$R_{1,2}$ & 1 & $\left(\begin{array}{c}1 / 2 \\
-1 / 2\end{array}\right)$ & 1 & 3 \\
$T$ & 1 & $2 / 3$ & 3 & 3 \\
$S$ & 1 & 0 & 1 & 3 \\
\hline \hline
\end{tabular}


group of the standard model (SM) is a subgroup of the unbroken $S U(8)$. The EFT description contains 63 pseudoNambu-Goldstone bosons (pNGBs), denoted as $\pi^{a}$, and one additional $S U(8)$ singlet, the dilaton, which we denote as $\chi$. We ignore the $U(1)_{A}$ meson, which is a singlet and has a large mass, due to the anomaly.

The CHM construction starts from the observation that eight of the pNGBs have the correct quantum numbers to form two copies of the Higgs doublet of the standard model. We further modify the dilaton EFT of Refs. [20-22] by adding two terms: a coupling of one of these two doublets to the top quark and a related potential term for the pNGBs. In this Letter, we ignore all SM fermions other than the top; the generalization to include other SM fermions within the EFT framework is straightforward.

The EFT Lagrangian density that results from this construction is the following:

$$
\begin{aligned}
\mathcal{L}= & \frac{1}{2}\left(\partial_{\mu} \chi\right)^{2}+\mathcal{L}_{\pi}+\mathcal{L}_{M}-V(\chi) \\
& +\mathcal{L}_{Y}-V_{t}+\mathcal{L}_{1},
\end{aligned}
$$

where the dilaton field $\chi$ acts as a conformal compensator, coupling to EFT operators in such a way as to restore scale invariance in Eq. (1). It acquires a $\operatorname{VEV~}\langle\chi\rangle \equiv F_{d}$, breaking scale invariance spontaneously.

The kinetic term for the pNGBs is

$$
\mathcal{L}_{\pi}=\frac{F_{\pi}^{2}}{4}\left(\frac{\chi}{F_{d}}\right)^{2} \operatorname{Tr}\left[D_{\mu} \Sigma\left(D^{\mu} \Sigma\right)^{\dagger}\right],
$$

where $F_{\pi}$ enters the EFT as the scale of spontaneous breaking of $S U(8) \times S U(8)$. The matrix-valued field $\Sigma$ represents the 63 pNGBs spanning the $S U(8) \times$ $S U(8) / S U(8)$ coset. The covariant derivatives describe couplings to the SM gauge bosons, following the embedding identified in Table I. Their kinetic terms and selfinteractions are the standard ones, which we include in $\mathcal{L}_{1}$. $\Sigma$ satisfies the nonlinear constraint $\Sigma \Sigma^{\dagger}=\mathbb{1}_{8}$.

The Dirac mass given to the fermions of the new strong sector leads directly to the following term in the EFT:

$$
\mathcal{L}_{M}=\frac{M_{\pi}^{2} F_{\pi}^{2}}{4}\left(\frac{\chi}{F_{d}}\right)^{y} \operatorname{Tr}\left[\Sigma+\Sigma^{\dagger}\right]
$$

and breaks the global symmetry. The quantity $M_{\pi}^{2}$ sets the scale for the masses of the 59 pNGBs besides those that become the Higgs doublet. The parameter $y$ has been interpreted as the scaling dimension of the fermion bilinear condensate in Ref. [27]. Its value is $y=2.06 \pm 0.05$ [22].

The scalar potential $V(\chi)$ describes the self-interactions of the dilaton field. It encodes both the spontaneous and explicit breaking of scale symmetry originating from the underlying gauge theory. We provided a general form for this potential in Ref. [22], where it played a key role.
Here we will not find it necessary to further invoke the explicit form of $V(\chi)$.

At the level of the EFT, we describe the mass of the top quark using the Yukawa interaction

$$
\mathcal{L}_{Y}=y_{t} F_{\pi}\left(\frac{\chi}{F_{d}}\right)^{z}\left(\bar{Q}_{L}^{\alpha} t_{R}\right) \operatorname{Tr}\left[P_{\alpha} \Sigma\right]+\text { H.c.. }
$$

The underlying gauge theory determines the scaling dimension $z . H_{\alpha} \equiv \operatorname{Tr}\left[P_{\alpha} \Sigma\right]$ transforms as the Higgs doublet, with quantum numbers $(2,-1 / 2)$ under $S U(2)_{L} \times U(1)_{Y}$. Here $\alpha$ is the index of $S U(2)_{L}$. We take the projectors $P_{\alpha}$ to be the following $8 \times 8$ matrices:

$$
P_{\alpha}=\left(\begin{array}{cc}
\tilde{P}_{\alpha} & \mathbb{O}_{4} \\
\mathbb{O}_{4} & \mathbb{O}_{4}
\end{array}\right),
$$

with

$$
\tilde{P}_{1}=\frac{1}{2}\left(\begin{array}{cccc}
0 & 0 & 1 & 0 \\
0 & 0 & 0 & 0 \\
0 & 0 & 0 & 0 \\
0 & -1 & 0 & 0
\end{array}\right), \quad \tilde{P}_{2}=\frac{1}{2}\left(\begin{array}{cccc}
0 & 0 & 0 & 0 \\
0 & 0 & 1 & 0 \\
0 & 0 & 0 & 0 \\
1 & 0 & 0 & 0
\end{array}\right) \text {. }
$$

This choice, motivated by simplicity, will ensure that one Higgs doublet is responsible for electroweak symmetry breaking.

The operator in Eq. (4) breaks the $S U(8) \times S U(8)$ global symmetry. In the underlying theory, interactions responsible for generating Eq. (4) also generate a $S U(8) \times S U(8)$ breaking contribution to the potential of the form

$$
V_{t}=-C_{t}\left(\frac{\chi}{F_{d}}\right)^{w} \sum_{\alpha=1}^{2}\left|\operatorname{Tr}\left[P_{\alpha} \Sigma\right]\right|^{2} .
$$

The (unknown) scaling dimension $w$ derives from the underlying gauge theory. This potential has non-Abelian global symmetry $S U(2)_{L} \times S U(2)_{R} \times S U(4)$, the $S U(4)$ remaining due to the vanishing entries in $P_{\alpha}$. The custodial $S U(2)_{R}$ symmetry, suppressing the effect of new physics on precision electroweak observables, is preserved here for our choice of $P_{\alpha}$, despite the fact that the Yukawa interaction in Eq. (4) breaks this symmetry explicitly. There are subleading contributions to this potential that break $S U(2)_{R}$, but they are smaller than $V_{t}$ and we will not consider them. Similarly, the gauging of the SM subgroup breaks the global symmetries and leads to additional contributions to the potential, but they are smaller and we neglect them in this analysis.

A loop of top quarks can also generate the interaction in Eq. (7), naturally making the constant $C_{t}$ positive. In addition, partial top compositeness can generate 
Eq. (4) —and hence Eq. (7)—in which case the field $Q_{L}^{\alpha}$ couples linearly to the baryon operator $\mathcal{B}_{L}^{\alpha}=L^{\alpha}\left(\overline{T R_{2}}\right)^{\dagger}+$ $R_{2}(\overline{T L})^{\dagger \alpha}$ and $t_{R}$ to $\mathcal{B}_{R}=2 R_{1}\left(T R_{2}\right)+L(T L)$ in the underlying theory. (Parentheses indicate contraction of spinor indices.) Alternatively, Eq. (4) can be generated by coupling the elementary fermion bilinear $\left(\bar{Q}_{L}^{\alpha} t_{R}\right)$ to the mesonic operator $\mathcal{O}_{M}^{\alpha}=\left(L^{\alpha} \bar{R}_{1}\right)-\epsilon^{\alpha \beta}\left(R_{2} \bar{L}_{\beta}\right)$.

The vacuum. - We first analyze the vacuum of the EFT. Both the pNGB that we identify with the Higgs field and the dilaton have nontrivial vacuum values, which must be calculated simultaneously. Then we determine the mass of the composite Higgs boson in this vacuum, emphasizing the significant role played by the dilaton field.

The three terms without derivatives or the top quark field in the Lagrangian of Eq. (1) define a potential for both the pNGBs and the dilaton. It is helpful to parametrize the $\Sigma$ field as

$$
\Sigma=\exp \left[i \theta\left(\begin{array}{ccc}
\mathbb{O}_{2 \times 2} & -i \mathbb{1}_{2} & \mathbb{O}_{2 \times 4} \\
i \mathbb{1}_{2} & \mathbb{O}_{2 \times 2} & \mathbb{O}_{2 \times 4} \\
\mathbb{O}_{4 \times 2} & \mathbb{O}_{4 \times 2} & \mathbb{O}_{4 \times 4}
\end{array}\right)\right],
$$

where only the degree of freedom corresponding to the pNGB component of the Higgs boson (represented by $\theta$ ) is shown, for simplicity. The potential then reads

$$
\begin{aligned}
W(\chi, \theta)= & V(\chi)-C_{t}\left(\frac{\chi}{F_{d}}\right)^{w} \sin ^{2} \theta \\
& -2 M_{\pi}^{2} F_{\pi}^{2}\left(\frac{\chi}{F_{d}}\right)^{y}(1+\cos \theta),
\end{aligned}
$$

Minimizing this potential determines the vacuum value $F_{d}$ of $\chi$, and the vacuum value of $\theta$ (the misalignment angle). We henceforth use $\theta$ to denote this vacuum value rather than the dynamical pNGB field. The electroweak scale $v \simeq 246 \mathrm{GeV}$ is related to the misalignment angle through $v=\sqrt{2} F_{\pi} \sin \theta$. The top acquires the mass $m_{t}=y_{t} v / \sqrt{2}$.

At the minimum of the potential, we have $\langle\chi\rangle \equiv F_{d}$, while

$$
\cos \theta=\frac{M_{\pi}^{2} F_{\pi}^{2}}{C_{t}}
$$

provided that $M_{\pi}^{2} F_{\pi}^{2}<C_{t}$; otherwise at the minimum we get $\theta=0$, preserving electroweak symmetry. Furthermore, the minimum must satisfy

$$
\begin{aligned}
0= & \left.\frac{\partial V}{\partial \chi}\right|_{F_{d}}-\frac{4 y M_{\pi}^{2} F_{\pi}^{2}}{F_{d}} \\
& -\frac{M_{\pi}^{2} F_{\pi}^{2}}{F_{d}}\left(w \frac{\sin ^{2} \theta}{\cos \theta}-2 y(1-\cos \theta)\right) .
\end{aligned}
$$

These equations determine $\theta$ in terms of $C_{t}$ and provide a relation between $F_{d}$ and other EFT parameters with the dilaton potential $V$.

To comport with the SM at currently accessible energies, we must find a small misalignment angle $\theta \ll 1$, that is, a large separation between $v$ and $F_{\pi}$. This is achieved by tuning $C_{t}$ in Eq. (10).

For $\theta \ll 1$, Eq. (11) determining $F_{d}$ simplifies in an essential way. The second line is suppressed and may be neglected in first approximation. The resulting equation is precisely the one used in Refs. $[20,21]$ to relate $F_{d}$ to the other parameters in the EFT employed there. That EFT, with no potential term proportional to $C_{t}$, was used to fit lattice data for the $S U(3)$ gauge theory with $N_{f}=8$. The functional form of the scalar potential $V(\chi)$ was constrained in that fit.

The mass matrix for the $\chi$ and $\theta$ degrees of freedom is approximately given (for small misalignment angle) by

$$
\mathcal{M}^{2}=\left(\begin{array}{cc}
M_{d}^{2} & \theta \frac{\sqrt{2} M_{\pi}^{2} F_{\pi}(y-w)}{F_{d}} \\
\theta \frac{\sqrt{2} M_{\pi}^{2} F_{\pi}(y-w)}{F_{d}} & \theta^{2} M_{\pi}^{2}
\end{array}\right),
$$

The $(1,1)$ entry is the second derivative of $W(\chi, \theta)$ with respect to $\chi$ at $\chi=F_{d}$ in the limit $\theta \rightarrow 0$. It is expressible in terms of the scalar potential $V(\chi)$ and other EFT parameters by

$$
M_{d}^{2}=\left.\frac{\partial^{2} V}{\partial \chi^{2}}\right|_{F_{d}}-4 y(y-1) \frac{F_{\pi}^{2}}{F_{d}^{2}} M_{\pi}^{2},
$$

which was employed in Refs. $[20,21]$ to fit lattice data. In the present context, $M_{d}$ is the approximate mass of the heavy scalar eigenstate, composed principally of $\chi$.

By diagonalizing the mass matrix in Eq. (12), we find that the mass $m_{h}$ of the lightest eigenstate (corresponding to the Higgs boson) is given by

$$
\frac{m_{h}^{2}}{v^{2}}=\frac{M_{\pi}^{2}}{2 F_{\pi}^{2}}\left(1-\frac{2 M_{\pi}^{2} F_{\pi}^{2}(y-w)^{2}}{M_{d}^{2} F_{d}^{2}}\right),
$$

up to $O(\theta)$ corrections. The second term in the large parentheses, arising from the presence of the dilaton field, is a distinctive feature of this model. Its presence allows us to accommodate the measured ratio $m_{h}^{2} / v^{2} \approx 0.26$, drawing directly on lattice data for which $M_{\pi}^{2} / 2 F_{\pi}^{2}$ is typically an order of magnitude larger.

Phenomenology.-In this section we examine the spectrum of particles of our CHM and the conditions under which constraints from collider experiments are satisfied. We also discuss the amount of fine-tuning needed for the model to satisfy the experimental constraints.

The spin 0 part of the spectrum of our EFT consists of 63 NGBs and pNGBs associated with the spontaneous breaking of the $S U(8) \times S U(8)$ symmetry of the underlying 
gauge theory, along with a scalar state of approximate mass $M_{d}$. The 3 massless NGBs are eaten by the $W^{ \pm}$and $Z$. One state is the relatively light pNGB Higgs boson of mass $m_{h}$ [Eq. (14)], while 59 are heavier pNGB states with their mass scale set by $M_{\pi}$. One additional heavier state has mass $M_{d}$. The quantities $F_{\pi}$ and $F_{d}$ are decay constants associated with these states. To set the relative size of $M_{\pi}, M_{d}$, $F_{\pi}$, we draw directly from the LSD lattice measurements [5].

Neglecting the SM gauge interactions, the EFT has approximate $S U(2)_{L} \times S U(2)_{R} \times S U(4)$ global symmetry. The pNGB multiplet decomposes into representations of this symmetry as follows:

$$
\begin{aligned}
63= & (3,1,1)+(1,3,1)+(1,1,1)+(2,2,1) \\
& +(1,1,15)+(2,1,4)+(1,2,4)+(1,1,1) .
\end{aligned}
$$

The misaligned vacuum breaks $S U(2)_{L} \times S U(2)_{R}$ spontaneously to its diagonal subgroup $S U(2)_{D}$. As a result, the composite spectrum is organized in a set of (approximate) multiplets of $S U(2)_{D} \times S U(4)$.

To determine the spectrum, we first specify the quantities $\left\{M_{\pi}^{2}, M_{d}^{2}, F_{\pi}^{2}, F_{d}^{2}, y, m_{h}, v\right\}$. We use data from lattice studies of the $N_{f}=8$ gauge theory. We extract the ratios $M_{\pi}^{2} / F_{\pi}^{2}$ and $M_{d}^{2} / F_{\pi}^{2}$ from Tables III and IV of Ref. [5] for five different constituent fermion masses $m_{f i}$. We then take $y=2.06 \pm 0.05$ and $F_{\pi}^{2} / F_{d}^{2}=0.086 \pm 0.015$ from Ref. [22] and set $m_{h}$ and $v$ to their experimentally determined values. Finally, we must set the overall scale for the new composite sector. As a benchmark, we take $M_{\pi}=4 \mathrm{TeV}$, to ensure that the 59 heavier pNGBs lie outside the reach of direct searches. The strongest bounds coming from searches for color octet scalars indicate that
$M_{\pi} \gtrsim 3.7 \mathrm{TeV}$ [57], although the precise bound depends on an additional coupling [58,59]. The calculated spectrum is then shown in Table II. It is nearly independent of which lattice point ( $m_{f i}$ value) we use in the analysis. The small variation in the mass of the heaviest singlet state (the dilaton) is mostly due to fluctuations in the lattice measurement of $M_{d}^{2} / M_{\pi}^{2}$.

In Table II we show only the central values for the masses of the spin 0 states, for the representative choices of parameters discussed previously, to illustrate the high degree of degeneracy among the heavier states, all of which have masses sitting within $15 \%$ of one another. The overall determination of the mass scale is affected by the large (and correlated) uncertainties originating in the lattice measurements of $M_{d}$ and $F_{\pi}$, and the indirect determination of $F_{d}$.

In calculating the spectrum, the quantities $w$ (for which there is no existing determination from lattice data) and $C_{t}$ are chosen to reproduce the aforementioned constraints. We find that $C_{t} \approx(2 \mathrm{TeV})^{4}$, with the precise determination depending on the lattice point considered. Similarly, $F_{\pi} \approx 1 \mathrm{TeV}$.

In Fig. 1, we show the impact of uncertainties in the lattice data on the allowed range for the scaling dimension $w$. For illustration purposes, we take lattice data for $M_{\pi}^{2} / M_{d}^{2}$ at the second fermion mass point $m_{f 2}$ from Ref. [5] (along with values for $y$ and $F_{\pi}^{2} / F_{d}^{2}$ from Ref. [22]) accounting for their uncertainties and shade in yellow the allowed ranges for $m_{h}^{2} / v^{2}$ and $w$. We see that if we require $m_{h}^{2} / v^{2} \simeq 0.26$ for consistency with experiment, $w$ could lie anywhere in the range $4.5<w<5.2$. Similar results hold for the other Table II values of the fermion mass $m_{f}$. If lattice simulations are able to measure $w$ with some precision

TABLE II. Estimated composite spectrum determined using the procedure described in the text. The states are labeled using their $S U(2)_{D} \times S U(4)$ quantum numbers, shown in the left-hand column. The $m_{f i}$ refer to the five different values for the constituent fermion mass appearing in the lattice study of Ref. [5] (arranged in ascending order) that is used as an input into these estimates. We show only the central values of the masses, to highlight the high level of degeneracy among the heavier states, which is independent of uncertainties coming from the lattice determinations of $M_{d}^{2}$ and $F_{\pi}^{2} / F_{d}^{2}$.

\begin{tabular}{lcccccc}
\hline \hline & \multicolumn{5}{c}{ Mass (TeV) } \\
\cline { 2 - 7 }$S U(2)_{D}$ & $S U(4)$ & $m_{f 1}$ & $m_{f 2}$ & $m_{f 3}$ & $m_{f 4}$ & $m_{f 5}$ \\
\hline 1 & 1 & 4.31 & 4.73 & 4.29 & 4.96 & 4.87 \\
3 & 1 & 4.35 & 4.37 & 4.39 & 4.40 & 4.40 \\
2 & 4 & 4.18 & 4.19 & 4.20 & 4.20 & 4.20 \\
3 & 1 & 4.03 & 4.03 & 4.04 & 4.04 & 4.04 \\
1 & 1 & 4.03 & 4.03 & 4.04 & 4.04 & 4.04 \\
1 & 1 & 4.03 & 4.03 & 4.04 & 4.04 & 4.04 \\
1 & 15 & 4.00 & 4.00 & 4.00 & 4.00 & 3.98 \\
1 & 1 & 3.99 & 3.98 & 3.98 & 3.98 & 3.82 \\
2 & 4 & 3.84 & 3.83 & 3.83 & 3.82 & 3.64 \\
3 & 1 & 3.67 & 3.66 & 3.64 & 3.64 & 0.126 \\
1 & 1 & 0.126 & 0.126 & 0.126 & 0.126 & 0 \\
3 & 1 & 0 & 0 & 0 & 0 & \\
\hline \hline
\end{tabular}




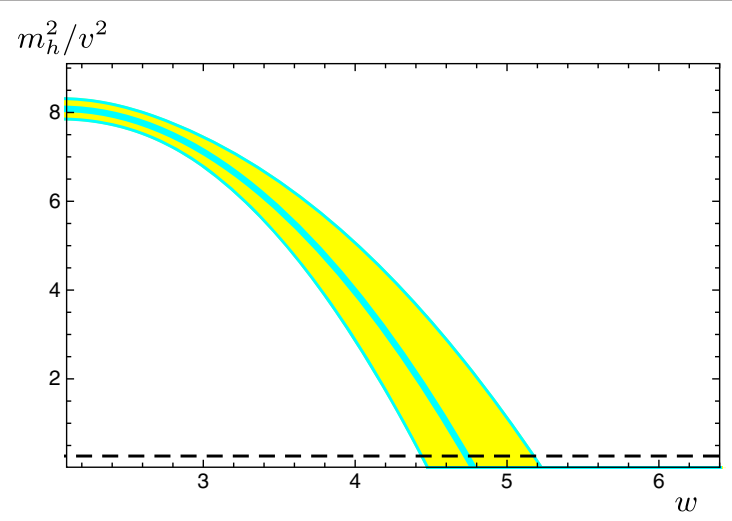

FIG. 1. The ratio $m_{h}^{2} / v^{2}$, as a function of $w$, for $y=2.06 \pm$ 0.05 and $F_{\pi}^{2} / F_{d}^{2}=0.086 \pm 0.015$ [22], for the LSD measurements taken at the second fermion mass $\left(m_{f 2}\right)$ in the range [5]. The horizontal black dashed line represents the experimental value $m_{h}^{2} / v^{2} \simeq 0.26$. The yellow shaded region is the uncertainty, which is dominated by the substantial uncertainty in the measurement of the mass $M_{d}$ of the scalar, and in the quantity $F_{\pi}^{2} / F_{d}^{2}$. For the best case scenario of $w=4.7$, the resulting uncertainty in $m_{h}^{2} / v^{2}$ is approximately an order of magnitude larger than the central value. Still, as the error in the measurement of $M_{d}$ is reduced, the range of acceptable $w$ values required to maintain $m_{h}^{2} / v^{2} \approx 0.26$ will diminish and potentially shift slightly.

(and measure $M_{\pi}^{2} F_{\pi}^{2} / M_{d}^{2} F_{d}^{2}$ with a similar precision), then meeting the requirement that $m_{h}^{2} / v^{2} \simeq 0.26$ could require an $m_{f}$ value outside the range of Table II.

Obtaining a spectrum with a realistic hierarchy $m_{h} \ll$ $M_{\pi}$ does require tuning $C_{t}$. For the parameter choices required to produce the spectrum in Table II, the misalignment angle satisfies $\theta \simeq 0.19$, implying a tuning for $C_{t}$ of order $2 \%$ through Eq. (10). The dilaton indirectly reduces the requisite tuning through Eq. (14): the effect of the term in parentheses (which depends on the dilaton) is to further suppress $m_{h}^{2} / M_{\pi}^{2}$, helping the colored pNGBs evade direct detection bounds.

Couplings between the Higgs and pairs of $W$ or $Z$ gauge bosons, as well as couplings between the Higgs and pairs of top quarks, deviate from their SM predictions in this CHM. These couplings include contributions from both the pNGB and dilaton components of the Higgs particle, and the expressions for them take the same form as those derived in the literature (see, e.g., Ref. [38] and references therein). In the limit $\theta \rightarrow 0$, the Higgs would couple to the gauge bosons and top with the same strength as the SM Higgs.

Using the benchmark $M_{\pi}=4 \mathrm{TeV}$ and the values for parameters selected by the lattice data of Ref. [5], we find that the ratio between the Higgs couplings to $W$ and $Z$ bosons and their SM values is approximately 0.98 . The coupling to top pairs has additional weak dependence on the unknown scaling dimension $z$, which arises because the dilaton component of the Higgs boson has a coupling to the top that is $z$ dependent from Eq. (4). For the value $z \simeq 2-3$, the coupling strength to the top becomes the same as in the
SM. Over a plausible range of values for $z$, the top coupling deviates from its SM value only by a few percent.

The amplitudes $h \rightarrow g g$ and $h \rightarrow \gamma \gamma$ also deviate slightly from their SM values. New electrically charged and colored pNGBs contribute to these amplitudes at the loop level, but are sufficiently heavy for our choice of benchmark that their contributions are negligible.

Considering all of these deviations, the signal significance for Higgs boson production in all observable channels would deviate from the SM prediction by no more than a few percent. Given the current accuracy of the Higgs measurements, which is no better than $8 \%$ [60], these effects will lie within experimental bounds, and a more precise analysis can be deferred.

The masses of composite states that are not included in the EFT have also been calculated in the $N_{f}=8$ gauge theory on the lattice in Ref. [5]. In particular, these data allow us to estimate the masses of the vector $(\rho)$ and axial $\left(a_{1}\right)$ mesons. Using lattice measurements for ratios $M_{\rho} / M_{\pi}$ and $M_{a_{1}} / M_{\pi}$, we estimate that the $\rho$ would have a mass in the 6-8 TeV range and the $a_{1}$ a mass in the $9-11 \mathrm{TeV}$ range, for our choice of benchmark $M_{\pi}=4 \mathrm{TeV}$. We therefore do not expect them to be detectable at the LHC. Given the small deviations in Higgs couplings, as well as the large $\rho$ and $a_{1}$ masses, precision electroweak observables such as the $S$ parameter will lie within current experimental bounds.

Summary.-We have argued that the $S U(3)$ gauge theory with $N_{f}=8$ fundamental fermions provides an attractive ultraviolet completion for a realistic composite Higgs model. This model has the distinctive feature that the near-conformal behavior of its underlying dynamics has been revealed by lattice studies.

We have drawn on such lattice results to compute several observable quantities within the model. These include the misalignment angle in the vacuum of the theory, the mass of the Higgs boson, and the spectrum of heavy scalars. We have also examined the Higgs boson couplings and its production rates at the LHC. The model passes all the direct and indirect tests currently available, at the price of a moderate amount of fine-tuning for one of the coefficients in the EFT potential.

We have described the model in terms of the dilaton EFT from Refs. [20-22], requiring only a simple addition to realize the Higgs doublet as composite pseudo-NambuGoldstone bosons. Because of the approximate scale invariance of the dilaton EFT, it is possible to accommodate a realistic value of the mass of the composite Higgs boson even for the values of the ratio $M_{\pi} / F_{\pi}$ currently available from lattice studies. As a consequence, the mass of the Higgs boson is suppressed by an order of magnitude with respect to that of the other pNGBs and dilaton in the EFT.

We look to future lattice studies for a determination of the scaling dimensions $z$ and $w$, which play important phenomenological roles. It will also be interesting to 
perform a more detailed study of the precision electroweak observables and explore the rest of the parameter space of this theory.

M.P. would like to thank G. Ferretti for useful discussion. The work of M. P. has been supported in part by the STFC Consolidated Grants No. ST/P00055X/1 and No. ST/T000813/1. M. P. has also received funding from the European Research Council (ERC) under the European Union's Horizon 2020 research and innovation programme under Grant Agreement No. 813942.

[1] Y. Aoki et al. (LatKMI Collaboration), Light composite scalar in eight-flavor QCD on the lattice, Phys. Rev. D 89, 111502 (2014).

[2] T. Appelquist et al., Strongly interacting dynamics and the search for new physics at the LHC, Phys. Rev. D 93, 114514 (2016).

[3] Y. Aoki et al. (LatKMI Collaboration), Light flavor-singlet scalars and walking signals in $N_{f}=8$ QCD on the lattice, Phys. Rev. D 96, 014508 (2017).

[4] A. D. Gasbarro and G. T. Fleming, Examining the low energy dynamics of walking gauge theory, Proc. Sci., LATTICE2016 (2017) 242.

[5] T. Appelquist et al. (Lattice Strong Dynamics Collaboration), Nonperturbative investigations of SU(3) gauge theory with eight dynamical flavors, Phys. Rev. D 99, 014509 (2019).

[6] Z. Fodor, K. Holland, J. Kuti, D. Nogradi, C. Schroeder, and C. H. Wong, Can the nearly conformal sextet gauge model hide the Higgs impostor?, Phys. Lett. B 718, 657 (2012).

[7] Z. Fodor, K. Holland, J. Kuti, S. Mondal, D. Nogradi, and C. H. Wong, Toward the minimal realization of a light composite Higgs, Proc. Sci., LATTICE2014 (2015) 244.

[8] Z. Fodor, K. Holland, J. Kuti, S. Mondal, D. Nogradi, and C. H. Wong, Status of a minimal composite Higgs theory, Proc. Sci., LATTICE2015 (2016) 219.

[9] Z. Fodor, K. Holland, J. Kuti, D. Nogradi, and C. H. Wong, The twelve-flavor $\beta$-function and dilaton tests of the sextet scalar, EPJ Web Conf. 175, 08015 (2018).

[10] Z. Fodor, K. Holland, J. Kuti, and C. H. Wong, Tantalizing dilaton tests from a near-conformal EFT, Proc. Sci., LATTICE2018 (2019) 196.

[11] Z. Fodor, K. Holland, J. Kuti, and C. H. Wong, Dilaton EFT from p-regime to RMT in the $\epsilon$-regime, arXiv:2002.05163.

[12] C. N. Leung, S. T. Love, and W. A. Bardeen, Spontaneous symmetry breaking in scale invariant quantum electrodynamics, Nucl. Phys. B273, 649 (1986).

[13] W. A. Bardeen, C. N. Leung, and S. T. Love, The Dilaton and Chiral Symmetry Breaking, Phys. Rev. Lett. 56, 1230 (1986).

[14] K. Yamawaki, M. Bando, and K. I. Matumoto, Scale Invariant Technicolor Model and a Technidilaton, Phys. Rev. Lett. 56, 1335 (1986).

[15] S. Matsuzaki and K. Yamawaki, Dilaton Chiral Perturbation Theory: Determining the Mass and Decay Constant of the Technidilaton on the Lattice, Phys. Rev. Lett. 113, 082002 (2014).
[16] M. Golterman and Y. Shamir, Low-energy effective action for pions and a dilatonic meson, Phys. Rev. D 94, 054502 (2016).

[17] A. Kasai, K. i. Okumura, and H. Suzuki, A dilaton-pion mass relation, arXiv:1609.02264.

[18] M. Hansen, K. Langaeble, and F. Sannino, Extending chiral perturbation theory with an isosinglet scalar, Phys. Rev. D 95, 036005 (2017).

[19] M. Golterman and Y. Shamir, Effective pion mass term and the trace anomaly, Phys. Rev. D 95, 016003 (2017).

[20] T. Appelquist, J. Ingoldby, and M. Piai, Dilaton EFT framework for lattice data, J. High Energy Phys. 07 (2017) 035.

[21] T. Appelquist, J. Ingoldby, and M. Piai, Analysis of a dilaton EFT for lattice data, J. High Energy Phys. 03 (2018) 039.

[22] T. Appelquist, J. Ingoldby, and M. Piai, Dilaton potential and lattice data, Phys. Rev. D 101, 075025 (2020).

[23] M. Golterman and Y. Shamir, Large-mass regime of the dilaton-pion low-energy effective theory, Phys. Rev. D 98, 056025 (2018).

[24] O. Cata and C. Muller, Chiral effective theories with a light scalar at one loop, Nucl. Phys. B952, 114938 (2020).

[25] M. Golterman, E. T. Neil, and Y. Shamir, Application of dilaton chiral perturbation theory to $N_{f}=8$, SU(3) spectral data, Phys. Rev. D 102, 034515 (2020).

[26] M. Golterman and Y. Shamir, Explorations beyond dilaton chiral perturbation theory in the eight-flavor SU(3) gauge theory, Phys. Rev. D 102, 114507 (2020).

[27] C. N. Leung, S. T. Love, and W. A. Bardeen, Aspects of dynamical symmetry breaking in gauge field theories, Nucl. Phys. B323, 493 (1989).

[28] A. G. Cohen and H. Georgi, Walking beyond the rainbow, Nucl. Phys. B314, 7 (1989).

[29] T. A. Ryttov and R. Shrock, Higher-loop corrections to the infrared evolution of a gauge theory with fermions, Phys. Rev. D 83, 056011 (2011).

[30] T. A. Ryttov and R. Shrock, Scheme-independent calculation of $\gamma_{\bar{\psi} \mu \psi, I R}$ for an SU(3) gauge theory, Phys. Rev. D 94, 105014 (2016).

[31] D. B. Kaplan and H. Georgi, $S U(2) \times U(1)$ breaking by vacuum misalignment, Phys. Lett. 136B, 183 (1984).

[32] H. Georgi and D. B. Kaplan, Composite Higgs and custodial SU(2), Phys. Lett. 145B, 216 (1984).

[33] M. J. Dugan, H. Georgi, and D. B. Kaplan, Anatomy of a composite Higgs model, Nucl. Phys. B254, 299 (1985).

[34] L. Vecchi, A dangerous irrelevant UV-completion of the composite Higgs, J. High Energy Phys. 02 (2017) 094.

[35] T. Ma and G. Cacciapaglia, Fundamental Composite 2HDM: SU(N) with 4 flavours, J. High Energy Phys. 03 (2016) 211.

[36] G. Panico and A. Wulzer, The composite Nambu-Goldstone Higgs, Lect. Notes Phys. 913, 1 (2016).

[37] O. Witzel, Review on composite Higgs models, Proc. Sci., LATTICE2018 (2019) 006.

[38] G. Cacciapaglia, C. Pica, and F. Sannino, Fundamental composite dynamics: A review, Phys. Rep. 877, 1 (2020).

[39] G. Ferretti, Gauge theories of partial compositeness: Scenarios for run-II of the LHC, J. High Energy Phys. 06 (2016) 107. 
[40] G. Cacciapaglia, G. Ferretti, T. Flacke, and H. Serôdio, Light scalars in composite Higgs models, Front. Phys. 7, 22 (2019).

[41] A. Hietanen, R. Lewis, C. Pica, and F. Sannino, Fundamental composite Higgs dynamics on the lattice: SU(2) with two flavors, J. High Energy Phys. 07 (2014) 116.

[42] W. Detmold, M. McCullough, and A. Pochinsky, Dark nuclei. II. Nuclear spectroscopy in two-color QCD, Phys. Rev. D 90, 114506 (2014).

[43] R. Arthur, V. Drach, M. Hansen, A. Hietanen, C. Pica, and F. Sannino, SU(2) gauge theory with two fundamental flavors: A minimal template for model building, Phys. Rev. D 94, 094507 (2016).

[44] R. Arthur, V. Drach, A. Hietanen, C. Pica, and F. Sannino, $S U(2)$ gauge theory with two fundamental flavours: Scalar and pseudoscalar spectrum, arXiv:1607.06654.

[45] C. Pica, V. Drach, M. Hansen, and F. Sannino, Composite Higgs dynamics on the lattice, EPJ Web Conf. 137, 10005 (2017).

[46] J. W. Lee, B. Lucini, and M. Piai, Symmetry restoration at high-temperature in two-color and two-flavor lattice gauge theories, J. High Energy Phys. 04 (2017) 036.

[47] V. Drach, T. Janowski, and C. Pica, Update on SU(2) gauge theory with $\mathrm{NF}=2$ fundamental flavours, EPJ Web Conf. 175, 08020 (2018).

[48] V. Ayyar, T. DeGrand, M. Golterman, D. C. Hackett, W. I. Jay, E. T. Neil, Y. Shamir, and B. Svetitsky, Spectroscopy of SU(4) composite Higgs theory with two distinct fermion representations, Phys. Rev. D 97, 074505 (2018).

[49] V. Ayyar, T. Degrand, D. C. Hackett, W. I. Jay, E. T. Neil, Y. Shamir, and B. Svetitsky, Baryon spectrum of SU(4) composite Higgs theory with two distinct fermion representations, Phys. Rev. D 97, 114505 (2018).

[50] V. Ayyar, T. DeGrand, D. C. Hackett, W. I. Jay, E. T. Neil, Y. Shamir, and B. Svetitsky, Finite-temperature phase structure of SU(4) gauge theory with multiple fermion representations, Phys. Rev. D 97, 114502 (2018).

[51] V. Ayyar, T. DeGrand, D. C. Hackett, W. I. Jay, E. T. Neil, Y. Shamir, and B. Svetitsky, Partial compositeness and baryon matrix elements on the lattice, Phys. Rev. D 99, 094502 (2019).

[52] G. Cossu, L. Del Debbio, M. Panero, and D. Preti, Strong dynamics with matter in multiple representations: SU(4) gauge theory with fundamental and sextet fermions, Eur. Phys. J. C 79, 638 (2019).

[53] E. Bennett, D. K. Hong, J. W. Lee, C.-J. D. Lin, B. Lucini, M. Piai, and D. Vadacchino, $\mathrm{Sp}(4)$ gauge theory on the lattice: Towards $\mathrm{SU}(4) / \mathrm{Sp}(4)$ composite Higgs (and beyond), J. High Energy Phys. 03 (2018) 185.

[54] E. Bennett, D. K. Hong, J. W. Lee, C.-J. D. Lin, B. Lucini, M. Piai, and D. Vadacchino, $\operatorname{Sp}(4)$ gauge theories on the lattice: $N_{f}=2$ dynamical fundamental fermions, J. High Energy Phys. 12 (2019) 053.

[55] E. Bennett, D. K. Hong, J. W. Lee, C. J. D. Lin, B. Lucini, M. Mesiti, M. Piai, J. Rantaharju, and D. Vadacchino, $S p(4)$ gauge theories on the lattice: Quenched fundamental and antisymmetric fermions, Phys. Rev. D 101, 074516 (2020).

[56] D. Buarque Franzosi, G. Cacciapaglia, and A. Deandrea, Sigma-assisted low scale composite GoldstoneHiggs, Eur. Phys. J. C 80, 28 (2020).

[57] A. M. Sirunyan et al. (CMS Collaboration), Search for high mass dijet resonances with a new background prediction method in proton-proton collisions at $\sqrt{s}=13 \mathrm{TeV}$, J. High Energy Phys. 05 (2020) 033.

[58] A. Belyaev, G. Cacciapaglia, H. Cai, G. Ferretti, T. Flacke, A. Parolini, and H. Serodio, Di-boson signatures as Standard Candles for Partial Compositeness, J. High Energy Phys. 01 (2017) 094.

[59] G. Cacciapaglia, A. Deandrea, T. Flacke, A. M. Iyer, GluonPhoton Signatures for color octet at the LHC (and beyond), J. High Energy Phys. 05 (2020) 027.

[60] G. Aad et al. (ATLAS Collabortaion), Combined measurements of Higgs boson production and decay using up to $80 \mathrm{fb}^{-1}$ of proton-proton collision data at $\sqrt{s}=13 \mathrm{TeV}$ collected with the ATLAS experiment, Phys. Rev. D 101, 012002 (2020). 